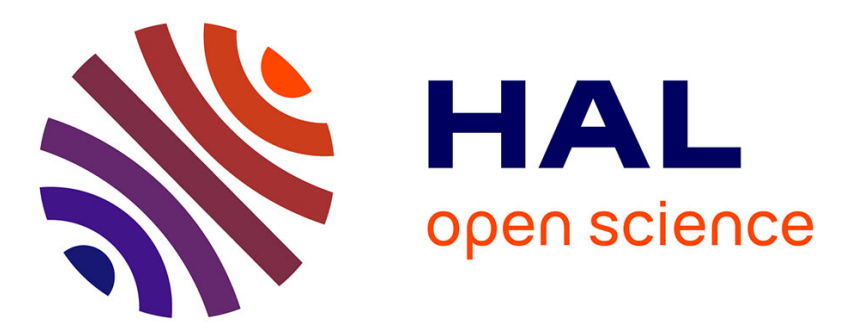

\title{
NATURE DES SAVOIRS EN SANTÉ. LE CAS DE L'ANNONCE D'UN DIAGNOSTIC DE MALADIE RARE
}

Séverine Colinet, Céline Avenel

\section{> To cite this version:}

Séverine Colinet, Céline Avenel. NATURE DES SAVOIRS EN SANTÉ. LE CAS DE L'ANNONCE D'UN DIAGNOSTIC DE MALADIE RARE. Savoirs : Revue internationale de recherches en éducation et formation des adultes, 2017, 10.3917/savo.045.0049 . hal-03288720

\section{HAL Id: hal-03288720 \\ https://hal.science/hal-03288720}

Submitted on 16 Jul 2021

HAL is a multi-disciplinary open access archive for the deposit and dissemination of scientific research documents, whether they are published or not. The documents may come from teaching and research institutions in France or abroad, or from public or private research centers.
L'archive ouverte pluridisciplinaire HAL, est destinée au dépôt et à la diffusion de documents scientifiques de niveau recherche, publiés ou non, émanant des établissements d'enseignement et de recherche français ou étrangers, des laboratoires publics ou privés. 


\title{
NATURE DES SAVOIRS EN SANTÉ. LE CAS DE L'ANNONCE D'UN DIAGNOSTIC DE MALADIE RARE
}

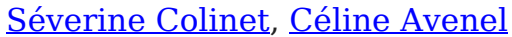

L'Harmattan | « Savoirs »

2017/3 N 45 | pages 49 à 66

ISSN 1763-4229

ISBN 9782343137759

Article disponible en ligne à l'adresse :

https://www.cairn.info/revue-savoirs-2017-3-page-49.htm

Distribution électronique Cairn.info pour L'Harmattan.

(c) L'Harmattan. Tous droits réservés pour tous pays.

La reproduction ou représentation de cet article, notamment par photocopie, n'est autorisée que dans les limites des conditions générales d'utilisation du site ou, le cas échéant, des conditions générales de la licence souscrite par votre établissement. Toute autre reproduction ou représentation, en tout ou partie, sous quelque forme et de quelque manière que ce soit, est interdite sauf accord préalable et écrit de l'éditeur, en dehors des cas prévus par la législation en vigueur en France. Il est précisé que son stockage dans une base de données est également interdit. 


\section{Nature des savoirs en santé. Le cas de l'annonce d'un diagnostic de maladie rare}

Séverine Colinet ${ }^{1}$ et Céline Avenel ${ }^{2}$

Résumé : Les compétences en situation professionnelle de l'annonce d'un diagnostic d'une maladie rare impliquant la mobilisation de savoirs par des médecins-praticiens, cet article se propose d'identifier la nature de ces savoirs mobilisés. Une enquête de terrain a été menée par entretiens semi-directifs auprès de 30 médecins rencontrés. Dans les récits d'expériences tenus par le groupe professionnel, la nature expérientielle et informelle des savoirs domine, à côté de savoirs de nature scientifique et procédurale. Cette première nature est révélatrice de résistances à une formalisation de l'annonce, et d'enjeux sur le plan de la formation. Cet article contribue à enrichir les travaux des sciences de l'éducation en santé, domaine en pleine expansion.

Mots-clés : savoirs de nature expérientielle-informelle/scientifique-procédurale, formation, médecins.

\section{Nature of knowledge in healthcare.} The case of announcing the diagnosis of a rare diseases

Considering competencies in the professional situation of medical practitioners announcing the diagnosis of a rare disease, implying the mobilization of the professional's knowledge, this article proposes to identify the nature of the knowledge thus mobilized. A field survey was conducted using semi-directive interviews with 30 physicians being questioned. In the professional group's narratives of their experiences, the experiential and informal nature of knowledge dominates along with scientific and procedural knowledge. This result reveals resistance to a formalization of the

1 Séverine Colinet est maitresse de conférences (BONHEURS) à l'Université de CergyPontoise. Ses recherches se situent au carrefour des champs de l'éducation et de la santé. Elles portent notamment sur la scolarisation des élèves hospitalisés, l'annonce diagnostique de maladies rares ou encore l'engagement associatif dans le cadre de parcours de santé.

2 Céline Avenel est docteure en sciences de l'éducation, post-doctorante (BONHEURS) à l'Université de Cergy-Pontoise. Ses recherches s'inscrivent dans le champ des sciences de l'éducation, et plus spécifiquement l'enseignement supérieur et la santé. Ses objets de recherche comprennent l'orientation dans le contexte des études de médecine, l'entrée dans la profession médicale et les pratiques d'annonce de la maladie rare. 
announcement and raises issues in terms of training. This article contributes to enriching the work of education sciences in the field of healthcare which is in full expansion.

Keywords: experiential-informal/scientific-procedural knowledge, training, doctors.

\section{Introduction}

Sur un plan légal, l'information au patient est un droit pour les personnes malades et les usagers du système de santé ainsi qu'une obligation pour les professionnels ou les établissements et services de santé (Article L1111-2 et L1111-4, Code de la santé publique). Pour Fraisse (2004), l'annonce d'un diagnostic grave est avant tout un «acte médical» de communication.

Il existe un début de tradition académique sur l'annonce. Les recherches en sciences sociales, en sociologie (Ebersold, 2007), en psychologie (Alvarez, Cayol, Magny et al., 2010 ; Rajon, Abadie et Grandjean, 2006) ou à l'initiative d'équipes médicales (Boucand, 2010) soulignent essentiellement les impacts psychologiques, identitaires (Cascales et Baruteau, 2012) et sociaux du diagnostic sur l'enfant (Drouin-Garraud, 2009). D'autres enquêtes font état de pratiques relatives au dévoilement du diagnostic par les professionnels (Pucci, Belardinelli, Borsetti et al., 2003). Les catégories d'analyse sont aussi liées aux différents types de pathologie qui font l'objet de l'annonce: la maladie d'Alzheimer (Novella, Blanchard, Drame et al., 2009), le cancer (Desportes et Spire, 2007), la maladie neuro-dégénérative (Mure Petitjean, Le Dastumer, Noel et al., 2007), la mucoviscidose (Langeard, Minguet, Guéganton et al., 2011), la pathologie psychiatrique (Galinowski, 2011). Chaque pathologie mobilise dans la situation d'annonce des postures et des attentes spécifiques vis-à-vis des patients.

Notre recherche porte sur l'annonce dans le cas d'une insuffisance rénale dont le fotus est atteint. Les formes les plus graves de cette maladie rare peuvent se déclarer précocement et la sévérité du diagnostic conduire certains couples à faire une demande d'interruption médicale de grossesse (IMG).

Les situations d'annonce sont complexes en raison de facteurs médicaux : l'incertitude relative au degré de l'insuffisance rénale dans l'élaboration du diagnostic, et sociaux : les représentations collectives de la maladie rénale ainsi que l'objet de l'interaction médicale. En effet : 
- certains aspects échographiques peuvent exister dans diverses anomalies rénales. Déterminer un diagnostic d'insuffisance rénale n'est pas systématiquement possible chez le fœtus ;

- le contexte de l'interaction médicale est spécifique : le médecin ne s'adresse pas directement au patient concerné par la pathologie puisqu'il s'adresse aux parents et non au fœtus ;

- les maladies rénales restent méconnues et les représentations culturelles sont confuses étant donné la possibilité de déclarer l'insuffisance à moyen ou long terme. Pour Desseix (2011), les maladies rénales souffrent de leur "petite renommée», le rein étant moins valorisé que d'autres organes vitaux comme le cœur. En outre, les patients connaissent généralement la pathologie du rein unique ou les traitements comme la dialyse et la greffe. Les représentations collectives génèrent ainsi l'idée que la maladie rénale est potentiellement bénigne, ce qui peut perturber la compréhension du diagnostic.

La complexité de l'annonce tient à ce qu'elle consiste à communiquer une information incertaine quant à l'évolution du fœtus dans un contexte de représentations paradoxales de la maladie chez les parents-patients, tout en incitant à la mise en place d'un accompagnement à la prise de décision de ces derniers.

Face à cette complexité, les praticiens sont amenés à mobiliser une compétence singulière associant une sélection des termes employés (pour ne pas réduire l'idée de l'enfant à naitre à l'unique imaginaire de la maladie), une attitude empathique et un discours compréhensible, tout en mobilisant les mots de la thérapie (comme la dialyse, la greffe) pour ne pas laisser les parents prendre leur décision en se représentant faussement l'anomalie de leur enfant à naître.

Les compétences en situation d'annonce impliquant la mobilisation par les médecins-praticiens de savoirs de nature différente, l'objectif de notre enquête exploratoire a été d'identifier la nature de ces savoirs. Pour cela, il s'est agi d'établir une photographie des récits d'expériences des médecins.

Après avoir présenté le cadre conceptuel de la recherche, nous exposerons le dispositif méthodologique mis en œuvre auprès des médecins. Nous analyserons dans leurs récits d'expériences la nature des savoirs mobilisés. Enfin, nous discuterons de la nécessité de développer une réflexion sur la formation médicale à l'annonce. 


\section{Cadre conceptuel}

\subsection{Sciences de l'éducation et santé}

Cette étude portant sur la nature des savoirs des médecins dans le contexte de l'annonce diagnostique s'inscrit dans la lignée des travaux en sciences de l'éducation sur les situations de soins.

Il existe déjà une tradition en sciences de l'éducation liée au champ de la santé (Tourette-Turgis et Thiévenaz, 2009; Dominicé et Jacquemet, 2014). En effet, on trouve des auteurs qui œuvrent pour une forte intégration des modèles de ces sciences dans les programmes dits d'éducation thérapeutique (Deccache et Aujoulat, 2001 ; d'Ivernois et Gagnayre, 2008). La communication médecin-malade également fait l'objet de théorisations. Des auteurs la rapprochent de l'éducation du patient (Fournier et Kerzanet, 2007) ou encore de sa formation thérapeutique (Lacroix et Assal, 2011), sous l'angle des habiletés communicationnelles et relationnelles (Richard, Lussier, Galarneau et al., 2010). D'autres travaux encore mettent en évidence la construction biographique de la maladie (Niewiadomski, 2000) ou s'attachent à l'adhésion du patient à la prise de décision (Debout, 2012).

L'annonce étant un sujet très ciblé, elle a suscité peu de travaux (Spire et Poinsot, 2007). En ce sens, ce travail est relativement innovant. Il s'inscrit dans le champ des sciences de l'éducation (Barbier, 2009 ; Lessard, Altet, Paquay et al., 2004 ), mais il vient également alimenter la sociologie des professions (Dubar, 2000) ou encore celle des professions médicales (Hughes, 1996 ; Ménoret et Carricaburu, 2004).

Afin de répondre à notre intention d'identifier la nature des savoirs mobilisés en situation professionnelle d'annonce, tentons de circonscrire les concepts de «compétences » et «savoirs » dont le pluriel souligne la diversité des types possibles.

\subsection{Compétences et mobilisation de savoirs}

\section{a) Compétences}

Le terme « compétence » est complexe car il rassemble un ensemble de notions : «posséder certaines connaissances, théoriques ou pratiques, avoir la capacité de mobiliser certains savoirs, certaines ressources dans l'action, avoir une bonne représentation de la situation, de ses performances... permet de construire des compétences » (Dolz et Ollagnier, 2002, p. 10).

Selon Le Boterf (2013), être compétent c'est « pour gérer des situations complexes et instables, savoir agir et savoir interagir» (Le Boterf, 2013, 
p. 65). La compétence se caractérise par ses dimensions individuelle (ressources personnelles, connaissances, savoir-faire, expériences) et collective (environnement, normes et règles du milieu professionnel).

L’approche situationnelle est également mise en évidence dans un certain nombre de définitions : "Les compétences ne sont pas une simple mise au travail de savoirs, d'opérations, de procédures apprises, mais une construction de réponses ajustées aux données situationnelles » (Sorel, 2008, p. 42). Selon Wittorski (1998), la compétence désigne « la mobilisation dans l'action d'un certain nombre de savoirs combinés de façon spécifique en fonction du cadre de perception que se construit l'acteur de la situation » (Wittorski, 1998, p. 60). Une compétence est alors conçue comme « un réseau intégré et fonctionnel constitué de composantes cognitives, affectives, sociales, sensorimotrices, susceptible d'être mobilisé en actions finalisées face à une famille de situations » (Allal, 2002, p. 81).

\section{b) Savoirs}

Pour Stroobants (1991), «le savoir perd son statut d'objet pour gagner en attribut du sujet [...] et la relation cognitive tend à se définir sur le mode de l'être (être compétent) et non plus sur celui de l'avoir (avoir un savoir au risque de le perdre)» (1991, p. 36).

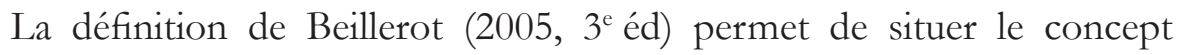
de «savoir» par rapport à celui d'«apprentissage »: «le savoir se définit comme ce qui, pour un sujet, est acquis, construit et élaboré par l'étude ou l'expérience. Résultat d'une activité d'apprentissage, quelles que soient la nature et la forme de celui-ci (imitation, imprégnation, identification, effet de l'action pédagogique, etc.), le savoir s'actualise dans des situations et dans des pratiques » (Beillerot, 2005, 3e éd., p. 898).

Selon Wittorski (1998), les savoirs portent une dimension sociale forte et peuvent être définis à la façon d'« un énoncé communicable socialement validé »(Wittorski, 1998, p. 61).

Il ne s'agit pas ici d'opposer compétence et savoir. En effet, «lorsque l'on oppose la compétence au savoir, la conception qui semble se dégager du savoir est celle d'un produit statique et figé alors que le savoir est dynamique » (Butlen et Dolz, 2015, p. 8).

De nombreux travaux se sont intéressés à la nature des savoirs, aux conditions de leur production (Lagadec, 2009) ou à leurs catégorisations (Perrenoud, 2005 ; Barbier, 2009). Mais aucun à notre connaissance n'a porté sur la nature des savoirs en situation d'annonce diagnostique. 


\section{Méthodologie}

Deux chercheurs en sciences de l'éducation ont réalisé cette étude.

Après approbation des instances éthiques ${ }^{3}$, une enquête par entretiens semi-directifs centrés (Merton, Fiske et Kendall, 1956) a été réalisée auprès de médecins pour recueillir des récits d'expériences d'annonce.

\subsection{Construction du guide d'entretien et déroulement de l'entrevue}

Pour le guide d'entretien, nous nous sommes inspirés de travaux portant sur les «récits de pratiques» (Bertaux, 1976) et l'entretien compréhensif (Kaufmann, 2011). Une collaboration entre chercheurs en sciences de l'éducation et médecins d'une équipe médicale nous a permis de l'élaborer. Il porte tout d'abord sur les éléments de cadrage: objet de la rencontre et respect des règles éthiques. Le récit d'expérience de l'annonce procède des thèmes principaux : « annonce en rapport avec une expérience particulière », « éléments les plus marquants de l'expérience », «vécu et ressenti », « informations sur le praticien : nombre d'années d'expérience, âge, sexe...».

Au moment de l'entretien, nous avons privilégié le «comment » plutôt que le "pourquoi », afin de saisir la nature des savoirs mobilisés et favoriser la production de sens pour le sujet interviewé : « comment avez-vous vécu cela ? ». Les relances reprenaient à l'identique la dernière phrase ou morceau de phrase de l'interlocuteur. Parfois nous reformulions, en questionnant: «comment expliquez-vous cela ?» pour inviter à développer le propos. Il arrivait aussi que nous restions silencieux pour privilégier l'auto-exploration de l'interlocuteur.

\subsection{Population de l'enquête}

Trente entretiens semi-directifs ont été réalisés auprès de médecins exerçant dans 20 services de 11 hôpitaux, échantillon composé de 15 femmes, âgées de 35 à 63 ans et de 45 ans en moyenne, et de 11 hommes, âgés de 38 à 60 ans et de 49 ans en moyenne.

Ces médecins sont issus de trois spécialités : la néphropédiatrie (16 médecins), la gynécologie/obstétrique (6 médecins) et la chirurgie pédiatrique (3 médecins). Nous avons rencontré à plusieurs reprises deux néphropédiatres avec qui nous avons évoqué différentes situations professionnelles d'annonce.

3 Comité consultatif sur le traitement de l'information en matière de recherche dans le domaine de la santé, le Comité de protection des personnes et la Commission nationale de l'informatique et des libertés. 
Le nombre d'entretiens de l'enquête est raisonné car nous avons veillé à prendre en compte une diversité de critères : géographie, anomalie médicale, genre et âge.

\subsection{Analyse des données}

Pour l'analyse, nous avons décidé de nous centrer sur les éléments de récit d'expérience des praticiens ayant trait à l'expression de la nature des savoirs.

Une première analyse du contenu thématique, inspirée de la théorie ancrée ou « Grounded Theory » (Glaser et Strauss, 2010), a eu pour objectif de dégager de façon inductive des catégories (Paillé et Mucchielli, 2013) en fonction des itérations de thèmes justifiant les représentations de l'annonce. Cette analyse a conduit à la construction de neuf catégories significatives qui classifient thématiquement les propos tenus (graphe $n^{\circ} 1$ ). Cette première phase a donné lieu à une grille d'analyse facilitant l'examen de chaque entretien. L'objectif était de comparer les catégories d'analyse (Bardin, 2003) présentes dans l'ensemble du corpus :

- premièrement, en prenant en compte le nombre de médecins ayant évoqué la catégorie ;

- deuxièmement, à partir du nombre d'occurrences de la catégorie et de ses sous-thèmes.

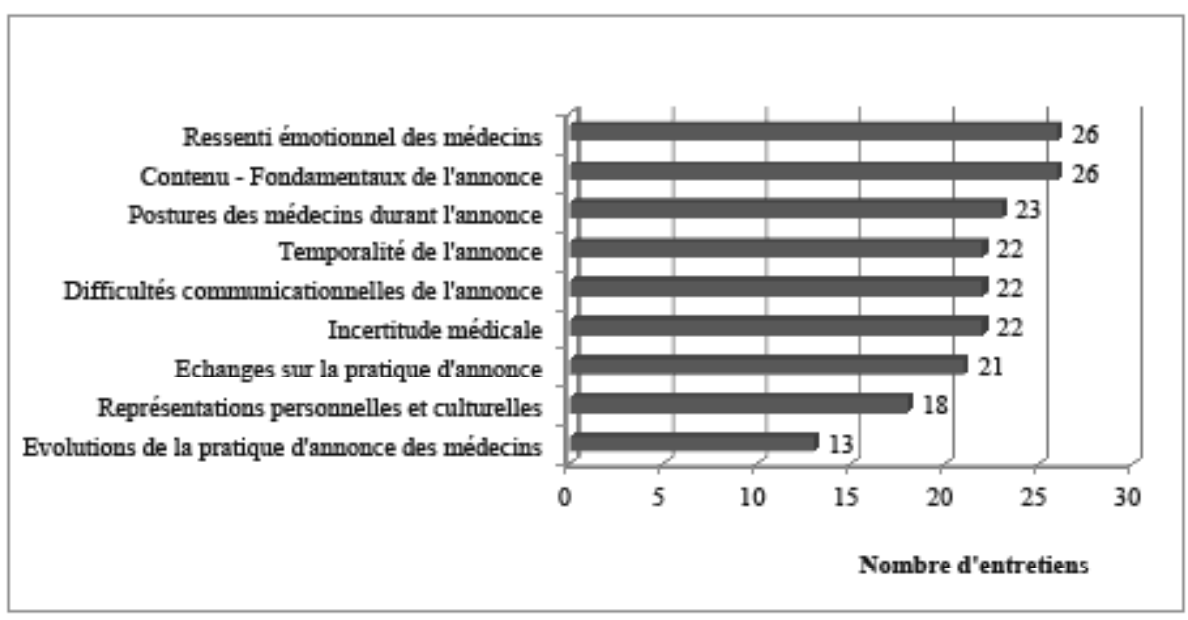

Graphe 1. Catégories significatives des récits d'expériences d'annonce issues de l'analyse du contenu thématique des entretiens avec les médecins

Une seconde analyse des récits d'expériences a été menée à l'aide du logiciel Tropes. Un double codage a été effectué : un premier où l'on a retenu 
la description de l'expérience d'annonce par le praticien, en éliminant les propos du chercheur et un second qui est une version condensée, sans les redondances encombrant la description d'une même situation.

Ce logiciel est fondé sur l'analyse cognitivo-discursive (Ghiglione, Landre, Bromberg et al., 1998). Il permet d'appréhender la catégorisation et l'agencement sémantique (Fallery et Rodhain, 2007) des propos tenus. Tropes construit des « références », ou thèmes, en ce sens qu’il comptabilise les champs lexicaux les plus utilisés par l'interlocuteur, et c'est de cette façon qu'ont pu être recensées par classe de fréquences les structures des récits.

L'objet de notre enquête n'était pas de dégager des résultats généralisables. Néanmoins, la robustesse des analyses a été obtenue par la création d'une série hiérarchisée de catégories et par une échelle contextuelle des verbatim.

\subsection{Limites de l'étude}

Les principaux biais méthodologiques de cette étude s'expliquent par l'absence de triangulation. Nous n'avons pas pu observer des consultations d'annonces ni participer à des réunions d'équipes de praticiens.

Par ailleurs, ayant souhaité, en tant que chercheurs en sciences de l'éducation, intervenir aux différentes étapes de l'étude pour une meilleure vue d'ensemble, nous n'avons pu disposer d'un comité d'analyse plus distancié par rapport à l'enquête réalisée. Une étude ultérieure pourrait pallier ces différentes faiblesses.

Le dispositif méthodologique nous a permis de dégager des résultats que nous allons à présent analyser.

\section{Résultats}

\subsection{Nature des savoirs des praticiens}

Pour caractériser la nature des savoirs, nous avons examiné la catégorie « contenu de l'annonce » apparue dans l'analyse de contenu thématique des récits d'expériences dans 26 entretiens de médecins/30. Trois sous-thèmes se dégagent : les étapes du discours, les facteurs contextuels et la perception du couple.

\section{a) Savoirs de nature scientifique et procédurale}

- La sous-catégorie «les étapes du discours » est présente chez près de 4 médecins sur 5, ce qui représente 400 occurrences, soit $42 \%$ de celles 
du thème «contenu ». Les médecins nous ont expliqué que l'annonce s'articule en phases classiques et systématiques, au sens de procédurales :

- le questionnement sur les antécédents médicaux du couple, les caractéristiques des familles ;

- la restitution des discours médicaux antérieurs ;

- l'information et l'explication des causes éventuelles, si le diagnostic est déjà établi à ce stade, de la maladie rénale du fotus.

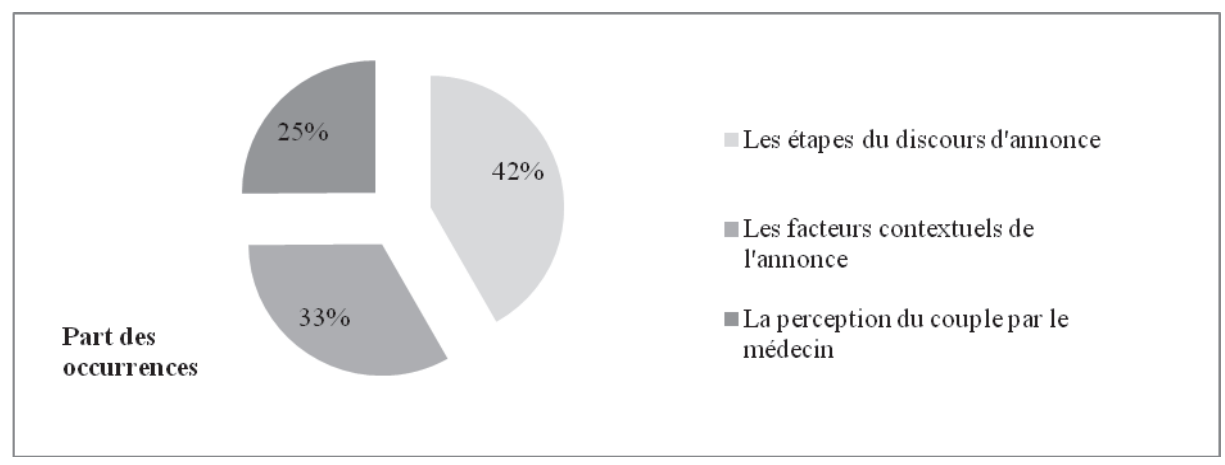

Graphe 2. Répartition détaillée par fréquences du thème «Contenu de l'annonce diagnostique »

Dans les récits d'expériences, les médecins font référence à un savoir de nature scientifique et procédurale. Selon Schön (1994), les savoirs scientifiques se fondent sur des modèles appris au cours de la formation, ce sont des savoirs enseignés. Ils procèdent de la démarche scientifique, c'est-à-dire des méthodes d'observation empirique du réel et de validation d'hypothèses (Perrenoud, 1996). Les savoirs procéduraux « précisent les normes, les précautions à prendre et disent dans quel ordre et avec quels moyens il faut faire les choses. Certains sont codifiés [...] (ou) utilisés tels qu'ils sont transmis par la culture professionnelle (Perrenoud, 1996, p. 242). Ils sont « formalisés et acquis en dehors de l'action » (Lenoir, Laforest et Pellerin, 1995, p. 114).

Ces étapes du discours occupent une place centrale dans le cursus de la formation initiale (Meyer et Cleary, 1998), dès le premier cycle préparant au Diplôme de formation générale en sciences médicales. Elles nécessitent des compétences cliniques, selon lesquelles les savoirs de nature scientifique et procédurale mobilisés consistent à : savoir mener l'anamnèse, savoir réaliser l'examen clinique, savoir identifier les examens complémentaires nécessaires, savoir synthétiser des données et savoir élaborer des hypothèses diagnostiques et des perspectives de prise en charge. Enseigner l'auto-examen (Kadaoui, 2012) est également évoqué quand le médecin aborde le futur parcours de soins de l'enfant. 
Au-delà de ces savoirs, l'analyse de contenu thématique montre que les récits d'expériences révèlent des savoirs de nature expérientielle et informelle.

\section{b) Savoirs de nature expérientielle et informelle}

Le sous-thème «les facteurs contextuels » représente $33 \%$ des occurrences, le sous-thème «la perception du couple» $25 \%$ des occurrences (cf. graphe 2). Ils occupent à eux deux $55 \%$ de la catégorie "contenu », soit plus de la moitié des occurrences des récits. Ces deux sous-thèmes font apparaitre des savoirs de nature expérientielle et informelle.

Vial, Caparros-Mencacci et Boyer (2004, p. 1) mentionnent des «savoirs de l'expérience informels » pour désigner des «savoirs acquis en situation, suite à un apprentissage non planifié, non institué ». Selon Courtois (2006), le savoir expérientiel est un "savoir local d'usage» et un "savoir insu», c'est-à-dire dépendant d'un contexte et difficile à formaliser, c'est un «savoir complexe multi-référentiel ».

Les facteurs contextuels ont été évoqués par 4 médecins sur 5, avec plus de 300 occurrences. Mais les praticiens en parlent de façon assez floue et la référence au contexte est mise en lien avec la variabilité et l'hétérogénéité des situations «c'est très variable en fonction du contexte, ses facteurs » (3-1-DD, chirurgien pédiatre). L'enquête souligne que les facteurs contextuels impliquent, sans aucune formalisation pour les praticiens, de savoir identifier des convictions personnelles, au point de se positionner parfois comme médiateurs dans l'échange, quand un facteur contextuel se surajoute au diagnostic médical. Ainsi ce médecin qui a été confronté à une discordance au sein du couple relative à une prise de décision d'IMG : «ce n'est vraiment pas simple, ils n'étaient pas d'accord. Et là ce n'était pas la conviction religieuse, c'était des convictions personnelles différentes et nous on est là en train de gérer le dialogue entre eux » (5-1-GD, néphrologue pédiatre). Ce propos révèle la dimension complexe de l'annonce.

La sous-catégorie "perception du couple» a été évoquée par plus de 4 médecins/5. Elle représente 226 occurrences de la catégorie « contenu». À partir des récits d'expériences, l'étude révèle que "percevoir, sentir le couple » revient à savoir détecter des signes, des comportements et des émotions manifestant une compréhension du diagnostic. En fonction de ces signes d'appréhension ou d'incompréhension, les praticiens mobilisent des compétences langagières (Kerbrat-Orecchioni et Cosnier, 1987 ; Grosjean et Lacoste, 1994 ; Mouthon et Hanslik, 2013) et plus spécifiquement une 
compétence d'adaptation : il s'agit de formuler le diagnostic dans un vocabulaire adapté au couple.

La perception consiste aussi à savoir où en est le couple de son cheminement quand la sévérité du diagnostic pose la question d'une éventuelle décision d'interruption de grossesse. Il s'agit de savoir prendre en compte les représentations sociales de la maladie et du corps malade chez les couples, discerner l'état émotionnel des parents afin de ne pas précipiter une décision d'interruption médicale de grossesse (IMG) et évaluer les signes attestant un cheminement des parents vers une décision comme, en particulier, le refus de l'IMG. L'extrait de récit ci-après témoigne de l'expérience d'un médecin face à un couple attendant son premier enfant à qui on a détecté une anomalie rénale. Ce couple se rendait pour la première fois à l'hôpital pour s'entretenir avec le néphropédiatre, après avoir rencontré un échographiste en ville : «Ils avaient l'air de comprendre ce que je disais. Je les ai sentis quand même... [...] ils ont encaissé quoi. On sentait que c'était pour eux... [...] Il faut sentir les émotions, c'est pas évident» (2-2-RS, néphrologue pédiatre, 51 ans). Là encore, la complexité de la situation est mentionnée.

Afin de mieux comprendre la mobilisation des savoirs de nature expérientielle et informelle, tentons d'identifier les facteurs explicatifs et les types de savoirs mobilisés.

\subsection{Les savoirs de nature expérientielle et informelle dans la situation d'annonce diagnostique : un consensus à l'origine de résistances à sa formalisation}

\section{a) Facteurs explicatifs}

Selon $3 / 4$ des médecins de l'enquête, l'annonce ne s'apprend pas en formation. Pour eux, il s'agit nécessairement d'apprendre à travers une accumulation d'expériences. Et selon l'enquête, l'annonce reste « séniorisée » et réservée aux titulaires. En outre, à travers l'usage d'outils identiques, poupées et schémas, pour rendre visible et concrète la prise en charge du futur enfant à naitre, on constate la mobilisation de savoirs « situés », c'est-à-dire dépendant de la socialisation professionnelle dans un service.

Les savoirs de nature expérientielle et informelle conduisent les médecins à refuser de remettre en question ce qui semble avoir le statut de valeurs communes dans la situation d'annonce: ne pas rompre l'intimité d'une consultation et son autonomie en faveur d'un travail normé et hiérarchiquement structuré. La complexité de l'annonce est alléguée pour justifier la 
nature des savoirs qui, en tant que produits de la socialisation professionnelle médicale, en freinent la formalisation.

\section{b) Les types de savoirs de nature expérientielle et informelle dans la situation d'annonce}

En nous inspirant du travail de classification de Cristol et Muller (2013), nous avons mis en correspondance les types de savoirs de nature expérientielle et informelle issus des analyses de notre enquête avec leurs principaux attributs.

\begin{tabular}{|l|l|}
\hline \multicolumn{1}{|c|}{$\begin{array}{c}\text { Types de savoirs } \\
\text { de nature expérientielle et informelle }\end{array}$} & \multicolumn{1}{c|}{ Principaux attributs } \\
\hline Par la perception & Rôle du ressenti comme facteur de savoirs \\
\hline Par l'autonomie & $\begin{array}{l}\text { Expérience qui justifie l'annonce, son autonomie } \\
\text { et l'intimité de la consultation }\end{array}$ \\
\hline Par la socialisation & $\begin{array}{l}\text { Rôle du groupe professionnel pour justifier la } \\
\text { non-formalisation de l'annonce }\end{array}$ \\
\hline «Situés » & $\begin{array}{l}\text { Effet-service : le lieu comme marqueur de } \\
\text { l'annonce }\end{array}$ \\
\hline
\end{tabular}

Tableau 1. Types de savoirs de nature expérientielle et informelle et leurs principaux attributs dans l'annonce diagnostique

Les résultats de l'enquête nous permettent à présent d'ouvrir la discussion.

\section{Discussion conclusive}

À l'issue de cette étude préliminaire, nous constatons que les praticiens mobilisent des savoirs de nature scientifique et procédurale d'un côté, de nature expérientielle et informelle de l'autre.

Nous avançons l'hypothèse d'une hétérogénéité de l'annonce chez les médecins, du fait de sa non-formalisation. Cela est dû à la conception personnelle, intime qu'ils en ont, en raison de leur mode de socialisation professionnelle. La norme commune est relative aux dimensions implicites et individuelles de l'annonce, laissant une large part aux savoirs de nature expérientielle et informelle. Mais cette norme participerait de l'invisibilité des savoirs, renforçant le sentiment de complexité ressenti par les médecins dans la situation d'annonce. À travers sa formalisation, il s'agirait donc d'harmoniser l'annonce, en lui donnant un cadre commun, afin de dépasser la part d'informel et d'individuel dans l'accompagnement parental, sans, bien entendu, gommer la singularité des situations. 
Ces éléments ouvrent sur de nouvelles perspectives. En s’inspirant du «renouveau pédagogique» (Parratte et Stip, 2012) proposé à l'Université de Montréal pour la formation en médecine, alors que l'approche des problèmes est essentiellement scientifique, les auteurs proposent un développement de l'approche par compétences. En ce sens, en France, il serait souhaitable de mettre en place, dès le premier cycle, en vue de l'obtention du Diplôme de formation générale en sciences médicales, une approche par compétences à l'annonce privilégiant l'acquisition de savoir-être pour ne pas les limiter à des savoirs informels, voire invisibles. Jusqu'ici le premier cycle est essentiellement consacré aux savoirs scientifiques (Fovet, Amad, Geoffroy et al., 2014). De même, lors du second cycle, le Diplôme de formation approfondie en sciences médicales (restructuré par l'arrêté du 8 avril 2013) sanctionne l'acquisition de savoirs médicaux généraux par le biais d'un enseignement à la fois théorique et pratique, où l'on retrouve les savoirs de nature scientifique et procédurale. Au niveau du TCEM (Troisième cycle des études médicales), l'interne reçoit une formation théorique et pratique comportant des fonctions hospitalières et extrahospitalières. Mais il est difficile d'assister aux entretiens cliniques, en stage, étant donné la croissance des effectifs de stagiaires dans les services (Fovet, Amad, Geoffroy et al., 2014). Or participer aux consultations d'annonce, c'est bénéficier d'un lieu et d'un temps de formation qu'il s'agirait de réaffirmer dans les référentiels et les programmes.

En outre, le développement de simulations au niveau de l'annonce, en tant que méthode de pédagogie active, pourrait être envisagé par un travail de mises en situation. Jusqu'ici, elles sont très présentes en médecine d'urgence ou en réanimation (Fovet, Amad, Geoffroy et al., 2014). Le programme Développement professionnel continu (Haute Autorité de Santé, 2016) mis en place en 2013 (Bras et Duhamel, 2008) offre également un espace pour réaffirmer la place de la formation à l'annonce comme obligation légale pour les médecins (Loi n 2009-879 du 21 juillet 2009). Il conviendrait donc, dans la formation initiale aussi bien que continue, de ménager des espaces de réflexivité sur les savoirs dans la situation d'annonce.

En fait, il existe un début de tradition et de formalisation des études portant sur les compétences relationnelles dans les situations d'apprentissage (Henry, Holmboe et Frankel, 2013) ou encore de soins (Formarier, 2007), de soins palliatifs et de mort (Von Gunten, Ferris et Emanuel, 2000) mais aussi de crise (Mauriac, Zeltner et Mallat, 2002). S’inscrivant dans cette lignée, notre enquête complète ces travaux. 


\section{Bibliographie}

Allal, L. (2002). « Acquisition et évaluation des compétences en situation scolaire ». In J. Dolz, E. Ollagnier (dir.) L'énigme de la compétence en éducation. Louvain-laNeuve : De Boeck, pp. 75-94.

Alvarez, L., Cayol, V., Magny, J.-F. et al. (2010). «L'ombre des traumatismes périnatales sur les premiers liens ». La psychiatrie de l'enfant, 53, 609-638.

Barbier, J.-M. (2009). « Voies pour la recherche en formation ». Éducation et didactique, 3(3), 120-129.

Bardin, L. (2003). L'analyse de contenu. Paris : PUF.

Beillerot, J. (2005). « Savoir ». In P. Champy, C. ÉtévÉ (dir.), Dictionnaire encyclopédique de l'éducation et de la formation. Paris : Nathan, p. 898.

Bertaux, D. (1976). Histoires de vie ou récits de pratiques? Méthodologie de l'approche biographique en sociologie. Paris : CORDES.

Boucand, M.-H. (2010). «Le diagnostic d'une maladie rare... perçu comme nomination, au risque d'une identification : une position fragile au monde». In E. Hirsh (dir.), Traité de bioéthique, Soigner la personne, évolutions, innovations thérapentiques. Toulouse : Erès, 310-322.

Bras, P.-L., Duhamel, G. (2008). Formation médicale continue et évaluation des pratiques professionnelles des médecins: rapport. Paris: Inspection Générale des Affaires Sociales.

Butlen, M., Dolz, J. (2015). «La logique des compétences : regards critiques ». Le français aujourd'bui, 191, 3-14.

Cascales, T., Baruteau, J. (2012). «Deuil de la maladie, une identité qui vacille». Cliniques, 4, 30-47.

Courtois, B. (2006). «La transformation de l'expérience : sens, savoirs, identités ». In H. Bézille, B. Courtois (dir.) Penser la relation expérience-formation. Lyon: Chronique Sociale, 89-101.

Cristol, D., Muller, A. (2013). «Les apprentissages informels dans la formation pour adultes ». Savoirs, 32, 11-59.

Debout, C. (2012). «Adhésion thérapeutique ». In M. Formarier, L. Jovic (dir.) Les concepts en sciences infirmières. Toulouse: Association de recherche en soins infirmiers, 50-53.

Deccache, A., Aujoulat, I. (2001). «A European perspective: common developments, differences and challenges in patient education ». Patient Education and Counseling, 44(1), 7-14.

Desportes, G., Spire, A. (2007). «Cancer, l'annonce du diagnostic ». Les Temps modernes, 642, 207-216.

Desseix, A. (2011). «L'hémodialyse, cette maladie. Approche anthropologique d'un amalgame ». Sciences sociales et santé, 29(3), 41-73. 
Dolz, J., Ollagnier, E. (2002). «La notion de compétence : nécessité ou vogue éducative». In J. Dolz, E. Ollagnier (dir.) L'énigme de la compétence en éducation. Louvain-la-Neuve : De Boeck Supérieur, 7-24.

Dominicé, P., Jacquemet, S. (dir.) (2009). « Formation et Santé ». Savoirs, 19(1), 7-36.

Drouin-Garraud, V. (2009). «Annonce diagnostique à l'enfant ». In S. Korf-Sausse (dir.), La vie psychique des personnes handicapées, ce qu'elles ont à dire, ce que nous avons à entendre. Toulouse : Erès, 75-87.

Dubar, C. (2000). La socialisation: construction des identités sociales et professionnelles. Paris : Armand Colin.

Ebersold, S. (2007). Parents et professionnels face au dévoilement du handicap. Toulouse : Erès.

Fallery, B., Rodhain, F. (2007). «Quatre approches pour l'analyse de données textuelles: lexicale, linguistique, cognitive, thématique ». Communication. $X V T^{e}$ Conférence de l'Association Internationale de Management Stratégique AIMS. Montréal.

Formarier, M. (2007). «La relation de soin, concepts et finalités ». Recherche en soins infirmiers, 89(2), 33-42.

Fournier, C., Kerzanet, S. (2007). «Communication médecin-malade et éducation du patient, des notions à rapprocher: apports croisés de la littérature ». Santé publique, 19(5), 413-425.

Fovet, T., Amad, A., Geoffroy, P.-A. et al. (2014). «État actuel de la formation des médecins généralistes à la psychiatrie et à la santé mentale en France ». L'Information psychiatrique, 90(5), 319-322.

Fraisse, P. (2004). «L'annonce d'un diagnostic grave par le pneumologue : un acte thérapeutique ». Maladies respiratoires, 21, 75-91.

Galinowski, A. (2011). «L'annonce du diagnostic en psychiatrie ». Laennec, 59, 44-58.

Ghiglione, R., Landre, A., Bromberg, M. et al. (1998). L'analyse automatique des contenus. Paris : Dunod.

Glaser, B., Strauss, A. (2010). La découverte de la théorie ancrée. Stratégies pour la recherche qualitative. Paris : Armand Colin.

Grosjean, M., Lacoste, M. (1994). Communication et intelligence collective. Le travail à l'hôpital. Paris : PUF.

Henry, S., Holmboe, E., Frankel, R. (2013). «Evidence-based competencies for improving communication skills in graduate medical education: a review with suggestions for implementation ». Medical Teacher, 35(5), 395-403.

Hughes, E. (1996). Le regard sociologique. Paris : Éditions de l'EHESS.

Ivernois, (d') J.-F., Gagnayre, R. (2008). Apprendre à éduquer le patient. Paris : Vigot. 
Kadaoui, N. (2012). « Pratiques médicales de dépistage du cancer du sein chez les femmes de 35 à 49 ans et 70 et plus ». Canadian Family Physician, 58(1), 39-46.

Kaufmann, J.-C. (2011). L'enquête et ses méthodes. L'entretien compréhensif. Paris: Armand Colin.

Kerbrat-Orecchioni, C., Cosnier, J. (dir.) (1987). Décrire la conversation. Lyon : PUL.

Lacroix, A., Assal, J.-P. (2011). L'éducation thérapeutique des patients. Accompagner les patients avec une maladie chronique : nouvelles approches. Paris : Maloine.

Lagadec, A.-M. (2009). «L'analyse des pratiques professionnelles comme moyen de développement des compétences : ancrage théorique, processus à l'œuvre et limites de ces dispositifs ». Recherche en soins infirmiers, 97, 4-22.

Langeard, C., Minguet, G., Guéganton, L. et al. (2011). « L’expérience professionnelle du médecin hospitalier à l'épreuve du dépistage: le cas de l'annonce du diagnostic de la mucoviscidose ». Revue francaise des affaires sociales, 2-3, 80-102.

Le Boterf, G. (2013). Construire les compétences individuelles et collectives. Le modèle : agir avec compétence en situation. Les réponses à plus de 100 questions. Paris : Eyrolles.

Lenoir, Y., Laforest, M., Pellerin, B. (1995). « Objets de formation privilégiés dans des curricula de formation des maîtres du primaire au Québec au cours des années quatre-vingt ». Spirale, 16, 107-135.

Lessard, C., Altet, M., Paquay, L. et al. (2004). Entre sens commun et sciences humaines : Quels savoirs pour enseigner? Bruxelles : De Boeck.

Mauriac, F., Zeltner, L., Mallat, V. (2002). «Intervenir en situation de crise». Enfances \& Psy, 18(2), 55-61.

Ménoret, M., Carricaburu, D. (2004). Sociologie de la santé. Institutions, professions, maladies. Paris : Armand Colin.

Merton, R., Fiske, M., Kendall, P. (1956). The focused interview. New York: The Free Press of Glencoe.

Meyer, J., Cleary, E. (1998). «An exploratory student learning model of clinical diagnosis ». Medical Education, 32(6), 574-581.

Mouthon, L., Hanslik, T. (2013). «L’observation médicale ». Collège national des enseignants de médecine Interne. Université médicale virtuelle francophone.

Mure-Petitjean, C., Le Dastumer, B., Noel, J.-L., Ankri J. (2007). « Pourquoi et comment donner une place à l'annonce diagnostique d'une maladie neurodégénérative au sein d'une consultation mémoire ? ». Gérontologie et société, vol. 30, $\mathrm{n}^{\circ} 121$, pp. 145-162.

Niewiadomski, C. (2000). Histoire de vie et alcoolisme. À la recherche d'un espace de construction de sens avec les personnes alcooliques. Paris : Seli Arslan.

Novella, J.-L., Blanchard F., Drame M. et al. (2009). «Le diagnostic précoce de la maladie d'Alzheimer modifie l'approche de la maladie ». Gérontologie et société, vol. 32, n $128-129$, pp. 177-182. 
Paillé, P., Mucchielli, A. (2013). L'analyse qualitative en sciences humaines et sociales. Paris : Armand Colin.

Parratte, J., Stip, E. (2012). «L'apprentissage par compétences: renouveau pédagogique à l'Université de Montréal ». L'Information psychiatrique, 88(7), 543-548.

Perrenoud, P. (1996). Enseigner: agir dans l'urgence, décider dans l'incertitude. Savoirs et compétences dans un métier complexe. Paris : ESF.

Perrenoud, P. (2005). «D’où viennent les savoirs mobilisés dans une analyse de pratiques ?». Intervention au $3^{\mathrm{e}}$ symposium des Groupes de Pairs. Société de Médecine Générale. Paris : 12 juin.

Pucci, E., Belardinelli, N., Borsetti, G. et al. (2003). «Relatives' attitudes towards informing patients about the diagnosis of Alzheimer's disease ». Journal of Medical Ethics, 29(1), 51-54.

Rajon, A.-M., Abadie, I., Grandjean, H. (2006). «Répercussions du diagnostic périnatal de malformation sur l'enfant et ses parents: approche métapsychologique à partir de l'étude longitudinale de 30 familles ». La Psychiatrie de l'enfant, 49, 349-404.

Richard, C., Lussier, M.-T., Galarneau, S. et al. (2010). «Compétence en communication professionnelle en santé ». Pédagogie médicale, 11, 255-272.

Schön, D. (1994). Le praticien réflexif : à la recherche du savoir caché dans l'agirprofessionnel. Montréal : Éditions Logiques.

Sorel, M. (2008). «À propos de la professionnalisation : le retour du sujet... ». Savoirs, 17(2), 37-50.

Spire, A., Poinsot, R. (2007). «L'annonce en cancérologie. Malades et maladies dans l'espace public ». Questions de communication, 11, 159-176.

Stroobants, M. (1991). "Travail et compétences: récapitulation critique des approches des savoirs au travail ». Formation et emploi, 33, 31-42.

Tourette-Turgis, C., Thiévenaz, J. (dir.) (2014). «L'éducation thérapeutique du patient ». Savoirs, 35(2), 9-48.

Vial, M., Caparros-Mencacci, N., Boyer, A. (2004). «La VAE à l'université : une occasion de prendre en considération des savoirs informels? ». Contribution. $7^{\mathrm{e}}$ Biennale de l'éducation et de la formation, pp. 1-6.

von Gunten, C., Ferris, F., Emanuel, L. (2000). «The patient physician relationship. Ensuring competency in end-of-life-care: communication and relational skills ». Journal of American Medical Association, vol. 23, n² 284, pp. 3051-3057.

Wittorski, R. (1998). «De la fabrication des compétences». Éducation permanente, 135, 57-69. 


\section{Textes de loi ou de cadrage et plan de santé publique}

Arrêté du 8 avril 2013 relatif au régime des études en vue du premier et du deuxième cycle des études médicales. Disponible sur Internet : <www.legifrance.gouv.fr/ affichTexte.do?cidTexte=JORFTEXT000027343762> (consulté le 31 août 2016).

Code de la santé publique. Article L1111-2. Disponible sur Internet: <www. legifrance.gouv.fr/affichCodeArticle.do?cidTexte=LEGITEXT0000060726 65\&idArticle $=$ LEGIARTI000006685758\&dateTexte $=\&$ categorieLien $=$ cid $>$ (consulté le 16 janvier 2016).

Code de la santé publique. Article L1111-4. Disponible sur Internet: <www. legifrance.gouv.fr/affichCodeArticle.do?idArticle=LEGIARTI000006685767 \&cidTexte=LEGITEXT000006072665 > (consulté le 15 janvier 2016).

Haute Autorité de Santé, Développement Professionnel Continu (DPC). Disponible sur Internet : <www.has-sante.fr/portail/jcms/c_1288556/fr/developpementprofessionnel-continu-dpc> (consulté le 15 janvier 2016).

Loi n² 2009-879 du 21 juillet 2009. Loi portant réforme de l'bôpital et relative aux patients, à la santé et aux territoires. Disponible sur Internet: <www.vie-publique. $\mathrm{fr} /$ actualite/panorama/texte-vote/loi-du-21-juillet-2009-portant-reformehopital-relative-aux-patients-sante-aux-territoires.html> (consulté le 17 janvier 2016). 